\title{
Correlation between Automated Perimetry and Optical Coherence Tomography in Post Treatment Follow-up of Papilledema due to Idiopathic Intracranial Hypertension
}

A.S.Beheiry, M.N.El-Mohamady, A.M.Saeed and T.M.Zaghloul

Ophthalmologist, Dept., Faculty of Medicine, Benha Univ., Benha, Egypt

E-mail: dramalbeheiry@gmail.com

\begin{abstract}
Objective: To assess correlation between functional changes of the optic nerve evaluated with automated perimetry (AP) and morphological changes of retinal nerve fiber layer (RNFL) evaluated with optical coherence tomography (OCT) in post treatment follow-up of papilledema due to idiopathic intracranial hypertension (IIH). Methods: We prospectively reviewed 40 eyes (20 patients) having recently diagnosed first attack of IIH with papilledema. Frisén grade of papilledema, Perimetric mean deviation (PMD) and spectral domain optical coherence tomography (SD-OCT) measurement of peripapillary retinal nerve fiber layer (RNFL) thickness were recorded at presentation and at $1^{\text {st }}, 3^{\text {rd }}$ and $6^{\text {th }}$ months after starting treatment. We excluded patients with papilledema due to causes other than IIH or glaucoma, and patients who were unwilling to give consent. Results: Our study included 20 patients (40 eyes). Age of patients ranged from 13 to 39 years (mean 27.55 \pm 8.4 years). Significant improvement of Frisén grade of papilledema was found throughout the study ( $\mathrm{p}<0.0001)$. No patients in the study showed worsening of papilledema grade through the follow up period. There was a significant improvement $(p<0.001)$ of the retinal nerve fiber layer (RNFL) thickness through the follow up in all patients. At presentation, the median papilledema grade was 2 (range 2-3), which dropped gradually through the study to reach a median of 1 (range 0-1) at the last visit. The mean PMD at presentation was $-2.05 \pm 7.25 \mathrm{~dB}$, improved gradually to reach a mean of $-0.59 \pm 4.02 \mathrm{~dB}$ at the last visit. RNFL thickness was $(176.7 \pm 89 \mu)$ at presentation, declined significantly $(\mathrm{p}<0.001)$ to $131.16 \pm 25.50$ then $121.57 \pm 14.20 \mu$ then $120.3 \pm 14.7 \mu$ at $1^{\text {st }}, 3^{\text {rd }}$ and $6^{\text {th }}$ month respectively. There was a significant correlation between PMD and RNFL thickness at the last visit $(\mathrm{p}=0.04)$. There was also a significant correlation $(\mathrm{p}<0.013)$ between Frisén grade of papilledema and RNFL thickness at all visits. We found that the correlation between papilledema grade and RNFL thickness $(\mathrm{r}=0.590)$ was stronger than the correlation between papilledema grade and PMD ( $r=-0.205)$ as shown by the correlation coefficient ( $r)$. We found no correlation between any of patient's age, laterality of the eye, oral contraceptive pills (OCP) intake or body mass index (BMI) and any of the study findings including best corrected visual acuity (BCVA), Frisén grade of papilledema, PMD or RNFL thickness. Conclusion: Our study showed a significant correlation between RNFL thickness and PMD at the end-of-study visit. Post treatment improvement of RNFL thickness was paralleled by recovery in PMD. As shown by the correlation coefficient $(r)$, we found that the correlation between papilledema grade and RNFL thickness $(r=0.590)$ was stronger than the correlation between papilledema grade and PMD ( $\mathrm{r}=-0.205)$.
\end{abstract}

Keywords: Papilledema, idiopathic intracranial hypertension, optic disc swelling, retinal nerve fiber layer, optical coherence tomography, visual field perimetry, enlarged blind spot.

\section{Patients and Methods}

This is a prospective clinical study that was carried out among candidate patients attending the Ophthalmology Department in Benha University Hospitals.

Inclusion criteria: The study included 20 patients (40 eyes) who all had a recently diagnosed papilledema due to first episode of IIH. Thorough discussion with every patient to explain the nature of the disease, goal of the study, and applied tests was done before obtaining an informed consent. Our study protocol followed all criteria of the Ethical Committee of Benha Faculty of Medicine.

Exclusion criteria: The study excluded patients with papilledema due to causes other than IIH as infection, cavernous venous sinus thrombosis, tumors and other space occupying lesions. Patients with chronic papilledema, neurological diseases, glaucoma, and patients who were unwilling to give consent were also excluded.

\subsection{Methods}

Personal history, past medical and ocular history, possible risk factors for IIH were taken. We assessed best corrected visual acuity (BCVA), intraocular pressure (IOP), color perception, pupillary reflexes, slit lamp biomicroscopy examination including assessment of papilledema grade, blood pressure check and body mass index (BMI) were recorded. All patients underwent automated perimetry in dim light to evaluate their visual field using SITA Standard 242 protocol by Humphrey Field Analyzer (Humphrey Field Analyzer I Carl Zeiss AG - Oberkochen Germany). However, in some visits of some cases, Oculus centerfield perimeter was used due to nonavailability of Humphrey Field Analyzer machine at the time of the visit. Spectral Domain OCT (SDOCT) imaging of optic nerve head was performed for assessment of peripapillary RNFL thickness using Topcon 3D-OCT 2000.

Treatment was delivered under supervision of both department of Ophthalmology and department 
of Neurology in Benha University Hospitals. Treatment included lifestyle modification advice, medical therapy, lumbar puncture (LP), and a plan to refer to shunt surgery whenever necessary. Overweight and obese patients were advised to have rest, avoid high-sodium foods and to undergo weight reduction regime under clinical supervision. Medical therapy included Acetazolamide (Cidamex $250 \mathrm{mg}$ ) tablets, Frusemide diuretic (Lasix $40 \mathrm{mg}$ tablets) and oral Potassium Chloride (Slow-K $600 \mathrm{mg}$ tablets), short course of steroid tablets in the form of prednisolone tablets when indicated. Lumbar puncture (LP) was performed, when necessary, as decided by the supervising neurologist and based upon severity of symptoms. Repetition of LP was done when symptoms persist or found intolerable at a two-week interval. All patients were consented about the procedure steps, possible complications and the possibility of repeating LP.

Treatment was started for all patients in the first visit. Then every patient was followed up at the $1^{\text {st }}$, $3^{\text {rd }}$ and $6^{\text {th }}$ months. At each follow-up visit, a complete ocular examination as well as assessment of severity of symptoms, papilledema grading, drug compliance and any possible adverse events were assessed and managed accordingly.

\section{Results}

Our study included 20 patients (40 eyes), all of them were females. Of note, three males were initially recruited in the study but were excluded later due to non-commitment to attend regular follow up visits. The study then included 20 female patients aged from 13 to 39 years (mean $27.55 \pm 8.4$ years). At time of presentation, all patients were experiencing IIH for the first time. Treatment started by ophthalmology and neurology departments in Benha University Hospital. Every patient was followed up at the $1^{\text {st }}, 3^{\text {rd }}$ and $6^{\text {th }}$ months.

During history taking, four female patients reported intake of oral contraceptive pills prior to the episode of IIH. No patients reported any other drug intake or previous episodes of IIH, no patients were suffering from any systemic diseases. No patients were smokers or ex-smokers, and none reported any history of known drug hypersensitivity reactions.

The main presenting symptom was Headache, reported by 14 patients $(70 \%)$, followed by vomiting that was reported by three patients $(15 \%)$ and was not related to meals, whilst blurred vision (5\%), vertigo as a single symptom (5\%), vertigo and tinnitus (5\%) were less reported. Four patients $(20 \%)$ were already on oral contraceptive pills before the baseline (first) visit. No patients were pregnant before the study, but four patients became pregnant between the third and the last visit. No patients had any past ocular history or previous ocular surgery. No patients had any history of systemic disease. Three patients (15\%) were known to have high body mass index $(\geq 30)$ throughout the study.

The visual acuity remained stable throughout the study. It remained around $0-0.2$ in LogMAR. No patients reported any drop of vision till the end of the study. The median LogMAR VA of right eyes was 0.00 and similarly for the left eye. No patients reported diminution of vision, impairment of color vision or pupillary response abnormalities. No patients showed elevation of the mean baseline IOP $(16 \mathrm{mmHg})$ along the study. There was no significant difference between right and left eyes in any of the measured parameters including BCVA, PMD, RNFL thickness, Frisén grade of papilledema or IOP.

The most common field defects in our study were enlargement of blind spot (EBS) with or without arcuate field defects. The least encountered field defect was the nasal field defect.

Frisén grade of papilledema ranged from grade 2 to grade 3 with median grade 2 at presentation. It declined to grade 2 (range 1.5-2) in $1^{\text {st }}$ month. Then to grade 1 (range $1-2$ ) at the $3^{\text {rd }}$-month. At the $6^{\text {th }}$ month visit, it declined to grade 1 (range 0-1). There was a significant decline of papilledema grade along the visits $(\mathrm{p}<0.0001)$. No patients in the study showed worsening of papilledema grade through the follow up period. There was an improvement of the PMD through the study from $-2.05 \pm 7.25 \mathrm{~dB}$ (at presentation) to $-0.59 \pm 4.02 \mathrm{~dB}$ (at last visit). All patients at presentation had above average RNFL thickness, with mean $176.7 \pm 89 \mu$ which dropped to $131.16 \pm 25.50 \mu$ at $1^{\text {st }}$ month follow up visit, then $121.57 \pm 14.20 \mu$ in the $3^{\text {rd }}$ month visit and to $120.3 \pm$ $14.7 \mu$ at the end of the study. This was a significant improvement in RNFL thickness ( $\mathrm{p}=0.001)$.

We found a significant correlation between PMD and RNFL thickness at the end of the study visit $(p=0.04)$. There was also a significant correlation between Frisén papilledema grade and RNFL thickness $(p<0.001)$. We found that the correlation between papilledema grade and RNFL thickness $(r=0.590)$ was stronger than the correlation between papilledema grade and PMD ( $\mathrm{r}=-0.205)$ as shown by correlation coefficient (r).

The mean perimetric mean deviation (PMD) in all eyes $(n=36)$ was $(-0.184 \pm 4.38 \mathrm{~dB}$. It was -0.742 $\pm 3.55 \mathrm{~dB}$ in the right eyes $(n=18)$ and $0.373 \pm 5.13$ $\mathrm{dB}$ in the left eyes $(\mathrm{n}=18)$. One patient did not attend the follow up visit. Two cases could not attend the last follow up visit. Another case missed the OCT visit as was feeling unwell.

The following figures show examples of papilledema grades, RNFL thickness, visual field changes and perimetric mean deviation in the four visits of the studied cases Figures (1-12). 


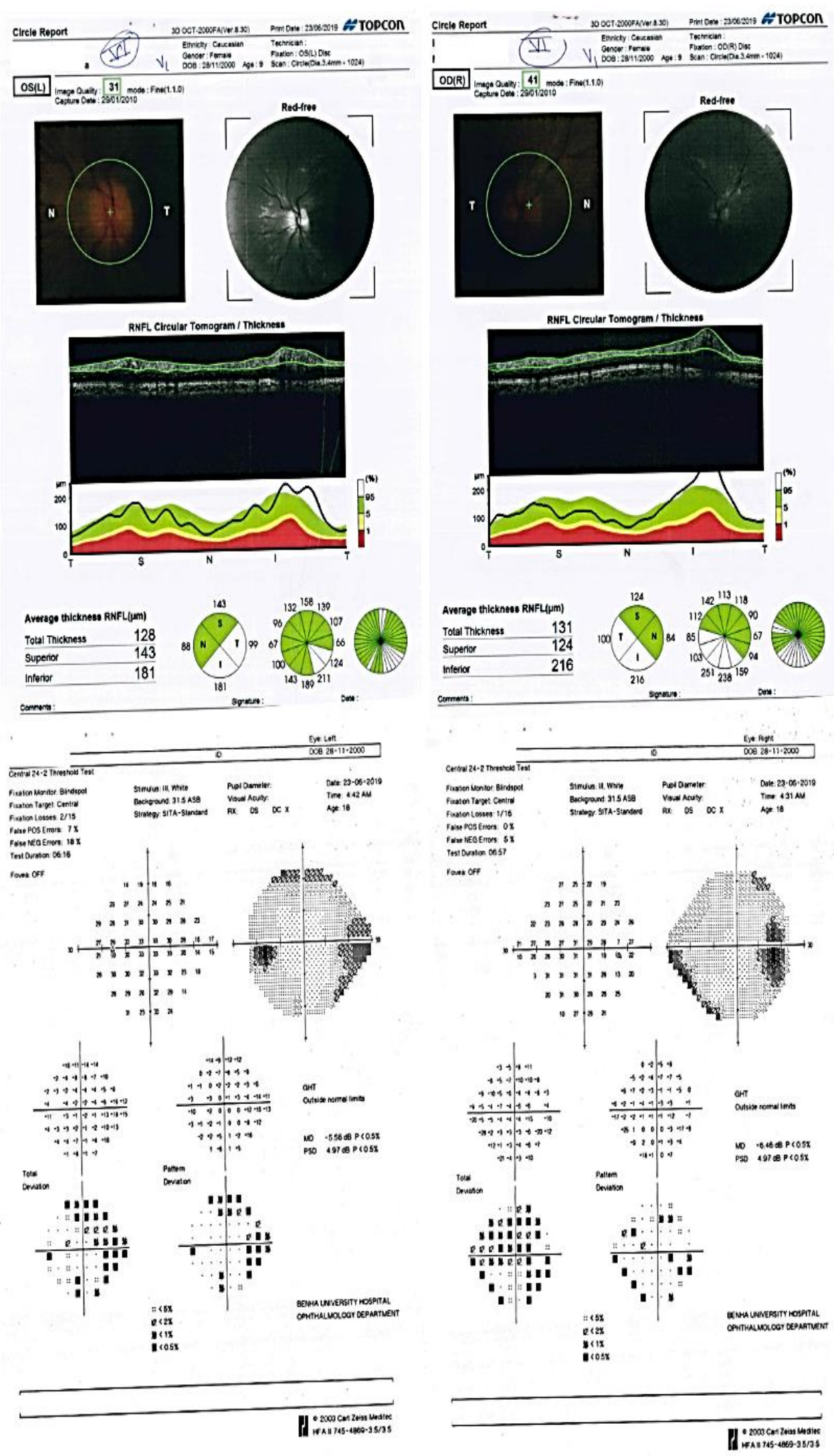

Fig. (1) OCT scan and VF test results of the first visit of patient number 6 in our study. Right eye: papilledema grade: 3. RNFL thickness: $131 \mu$, Visual field findings are EBS, arcuate scotoma in lower nasal field, early nasal defect. $\mathrm{MD}=-6.46 \mathrm{~dB}$. Left eye: papilledema grade 2 . RNFL thickness: $128 \mu$. Visual field findings are EBS, upper and lower incomplete arcuate scotomas, nasal field defect. $\mathrm{MD}=-5.56 \mathrm{~dB}$. 

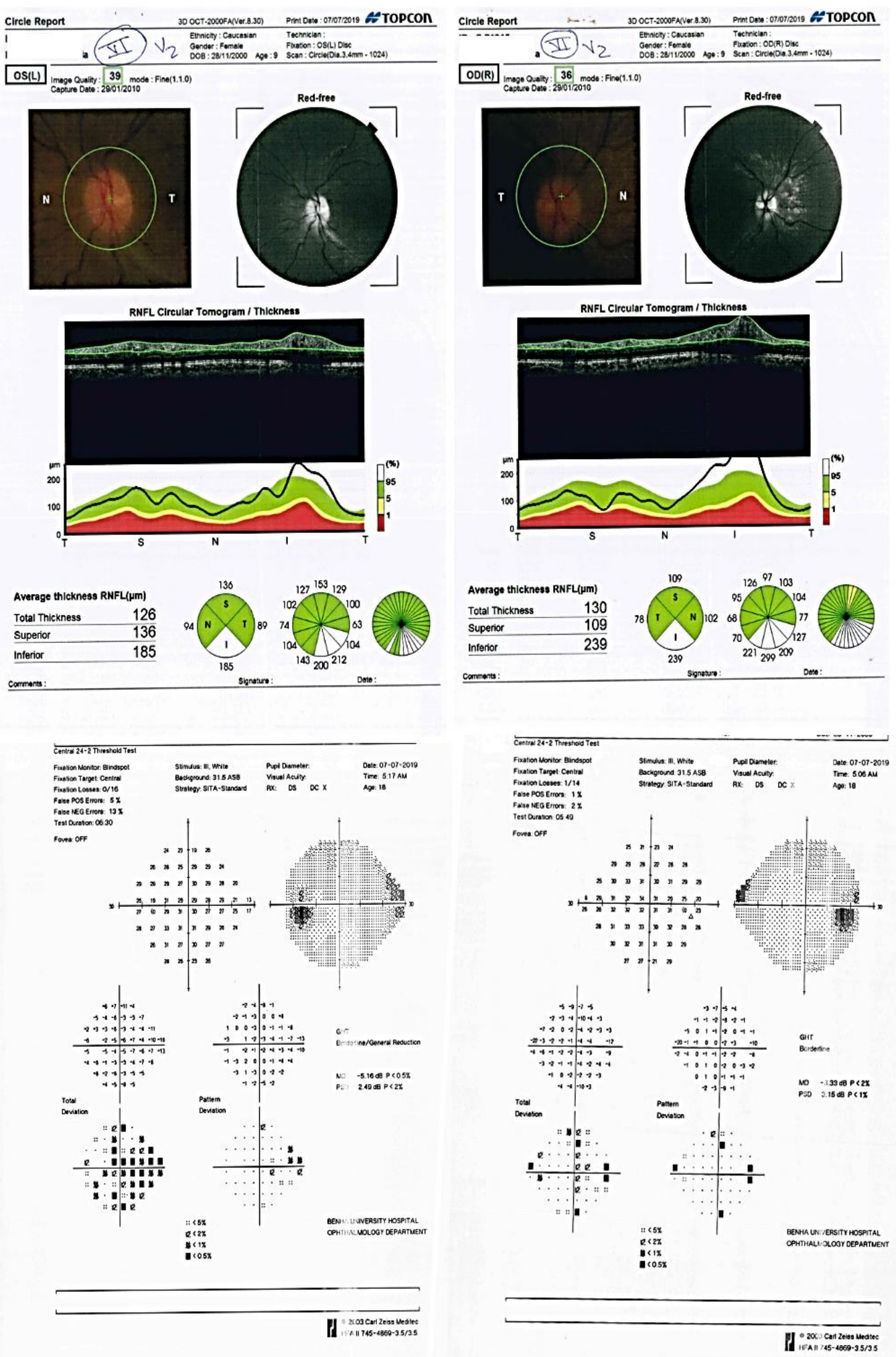

Fig. (2) OCT scan and VF test results of the second visit of patient number 6 in our study. Right eye: papilledema grade: 3 . RNFL thickness: $130 \mu$. Visual field findings are EBS, few scattered scotomas. MD = -3.33 dB. Left eye: papilledema grade 2. RNFL thickness: $126 \mu$. Visual field findings are nasal field defect. MD $=-5.16$ $\mathrm{dB}$. 

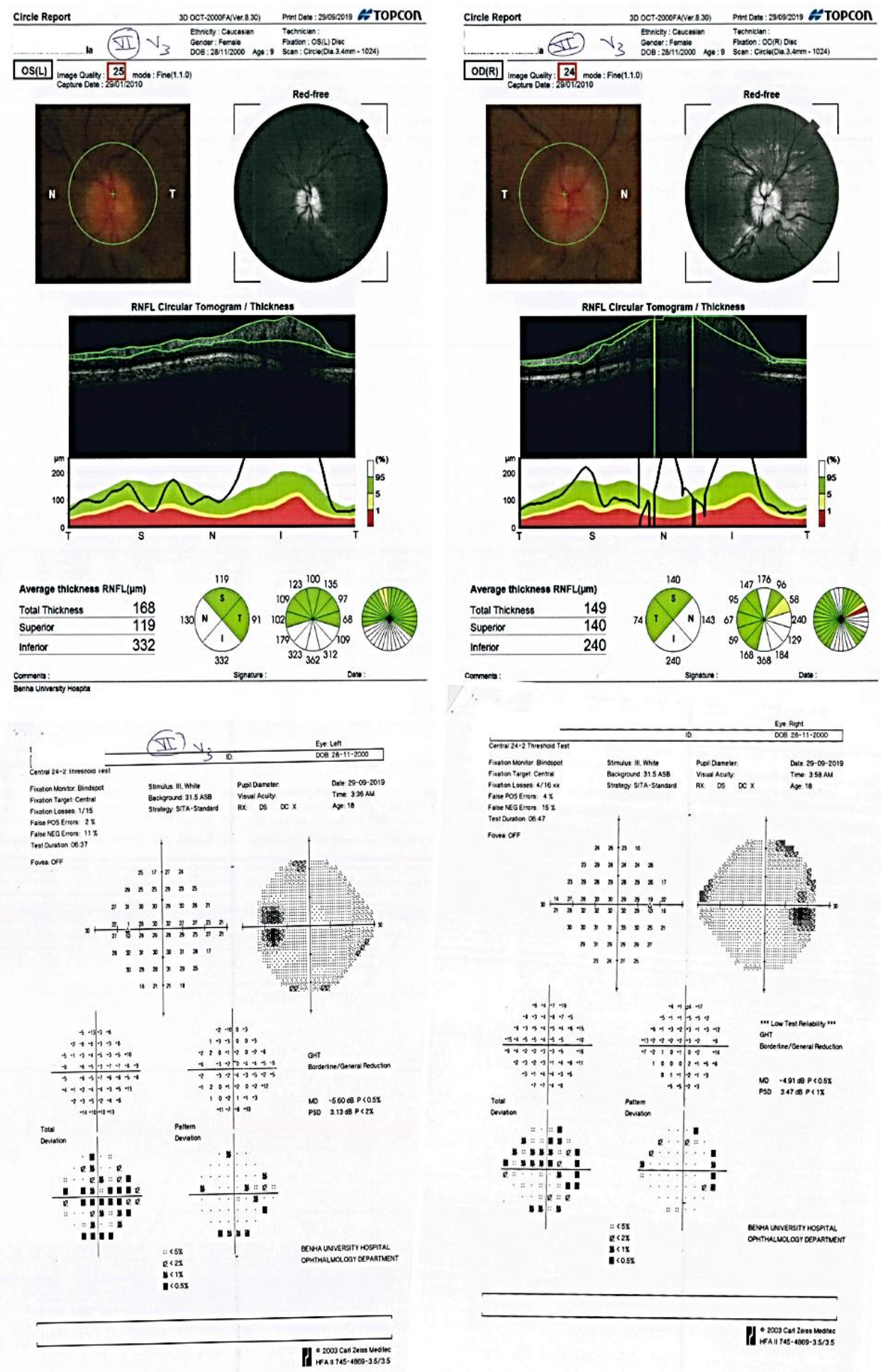

Fig. (3) OCT scan and VF test results of the third visit of patient number 6 in our study. Right eye: papilledema grade: 2. RNFL thickness: $149 \mu$, Visual field findings are EBS, superior incomplete arcuate scotoma. $\mathrm{MD}=-4.91 \mathrm{~dB}$. Left eye: papilledema grade 2. RNFL thickness: $168 \mu$. Visual field findings are EBS, nasal and inferior field defects. $\mathrm{MD}=-5.60 \mathrm{~dB}$ 


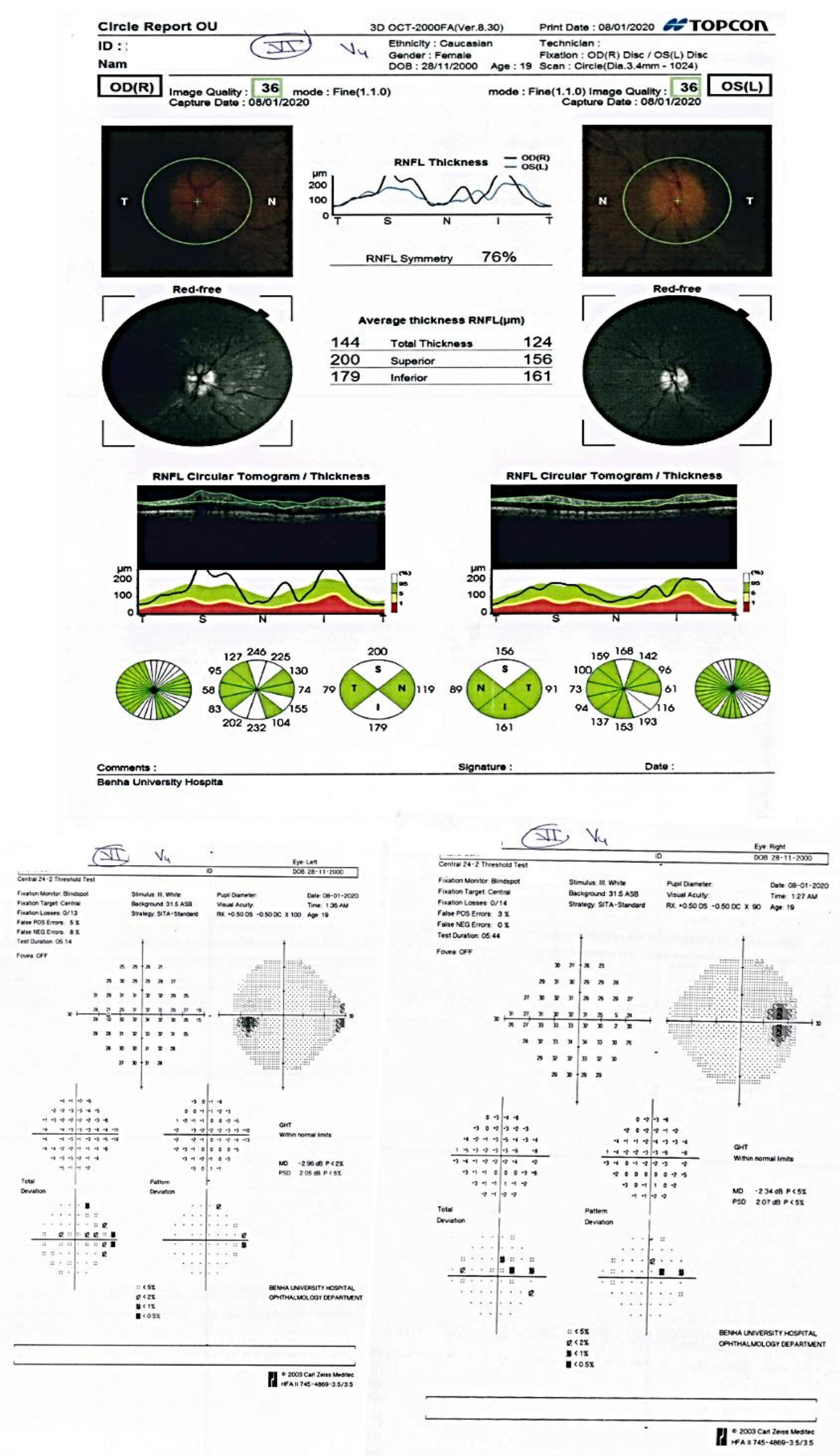

Fig. (4) OCT scan and VF test results of the fourth visit of patient number 6 in our study. Right eye: papilledema grade: 1 . RNFL thickness: $144 \mu$, Visual field finding is EBS. MD = $-2.34 \mathrm{~dB}$. Left eye: papilledema grade 1. RNFL thickness: $124 \mu$. Visual field findings are few nasal field defects. $\mathrm{MD}=-2.96 \mathrm{~dB}$. 

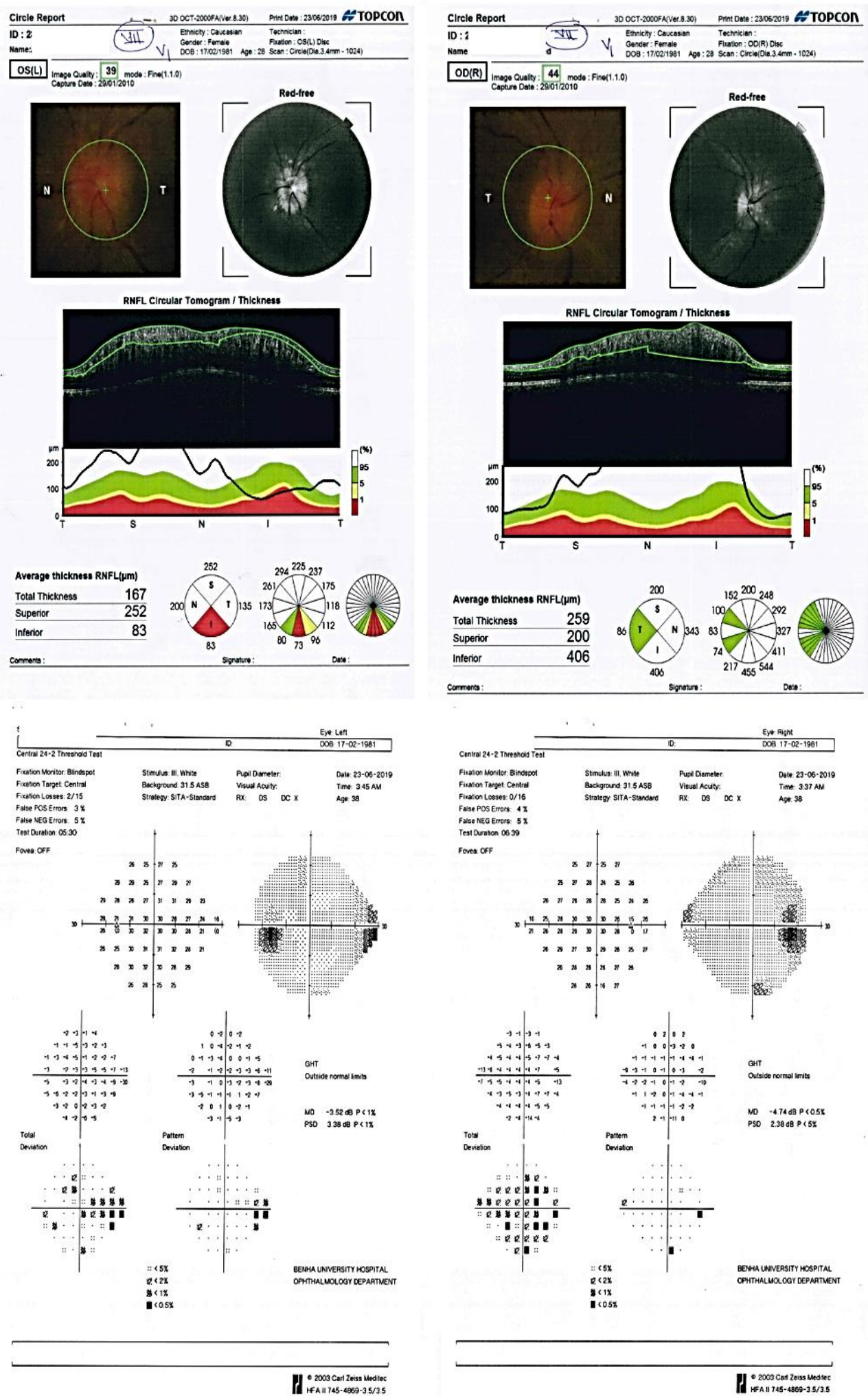

Fig. (5) OCT scan and VF test results of the first visit of patient number 7 in our study. Right eye: papilledema grade: 2. RNFL thickness: $259 \mu$, Visual field finding are EBS and few scattered non adjacent scotomas. MD $=-4.74$ dB. Left eye: papilledema grade 2. RNFL thickness: $167 \mu$. Visual field findings are EBS and small nasal field defect. $\mathrm{MD}=-3.52 \mathrm{~dB}$.. 

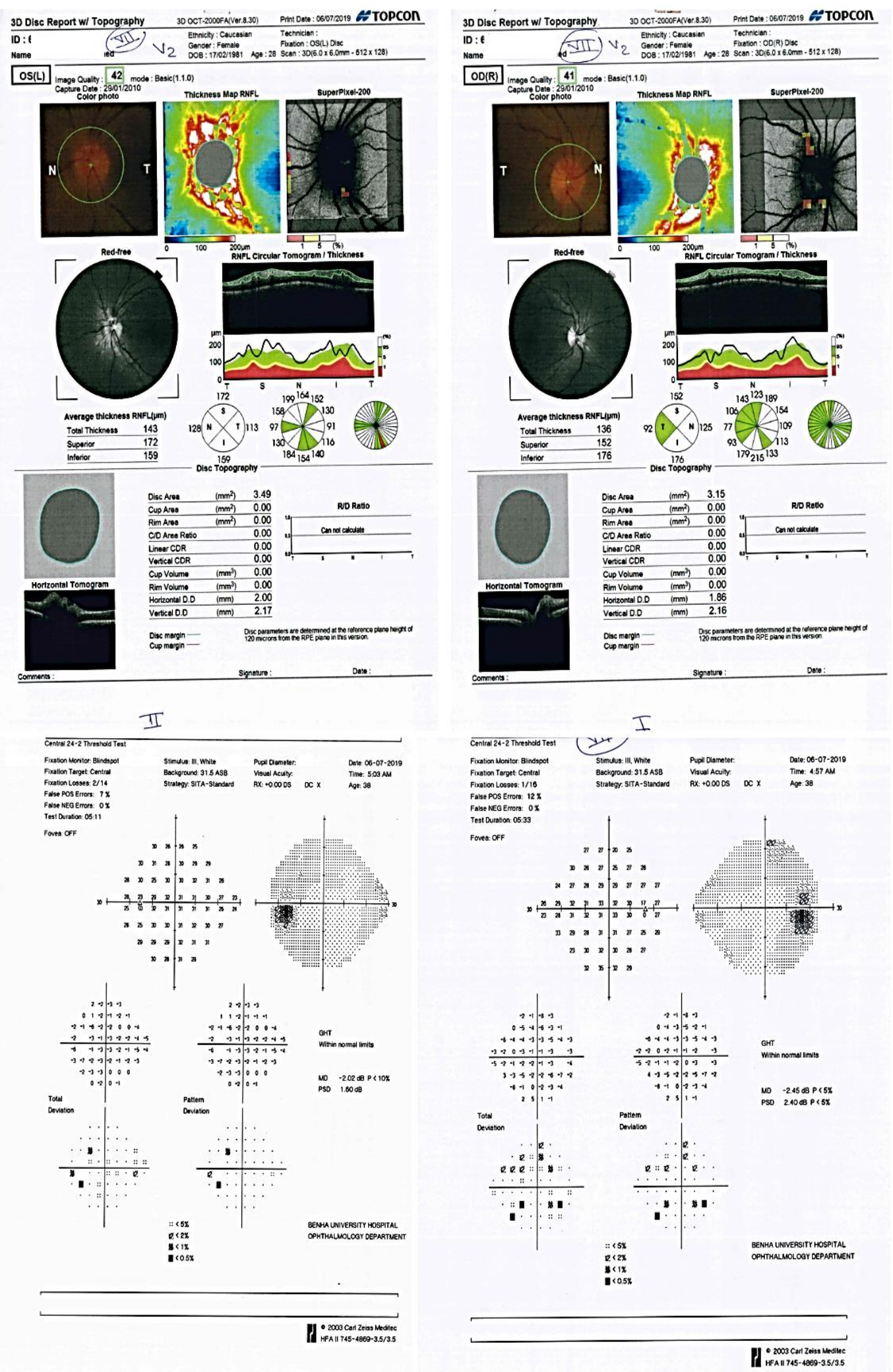

Fig. (6) OCT scan and VF test results of the second visit of patient number 7 in our study. Right eye: papilledema grade: 2 . RNFL thickness: $136 \mu$, Visual field finding is EBS and scattered superior and inferior scotomas. $\mathrm{MD}=-2.45 \mathrm{~dB}$. Left eye: papilledema grade 2 . RNFL thickness: $143 \mu$. Visual field finding is EBS. MD = $-2.02 \mathrm{~dB}$. 

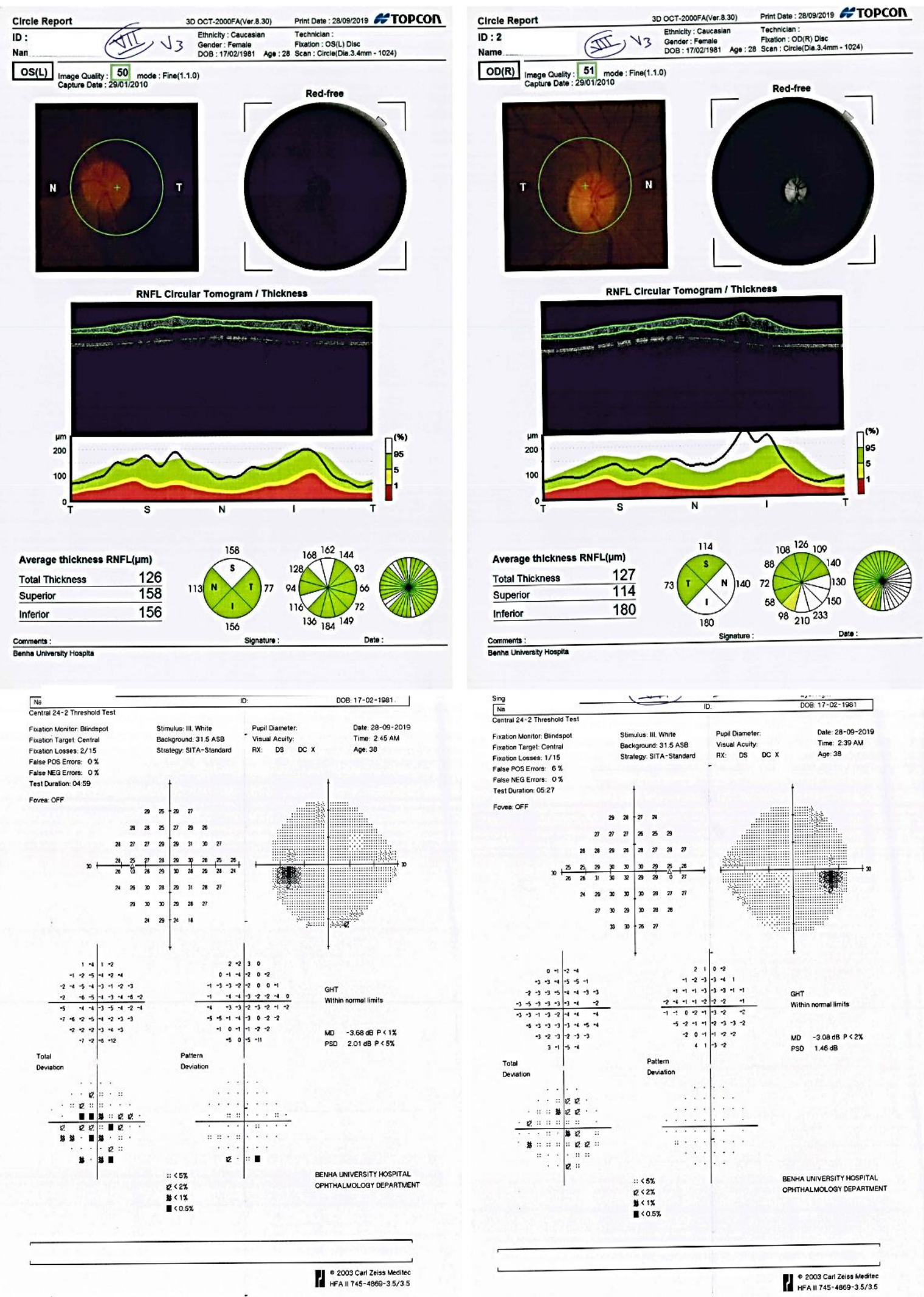

Fig. (7) OCT scan and VF test results of the third visit of patient number 7 in our study. Right eye: papilledema grade: 1 . RNFL thickness: $127 \mu$, Visual field finding MD $=-3.08 \mathrm{~dB}$. Left eye: papilledema grade 2 . RNFL thickness: $126 \mu$. Visual field findings are 2 inferior non adjacent scotomas. $\mathrm{MD}=-3.68 \mathrm{~dB}$. 

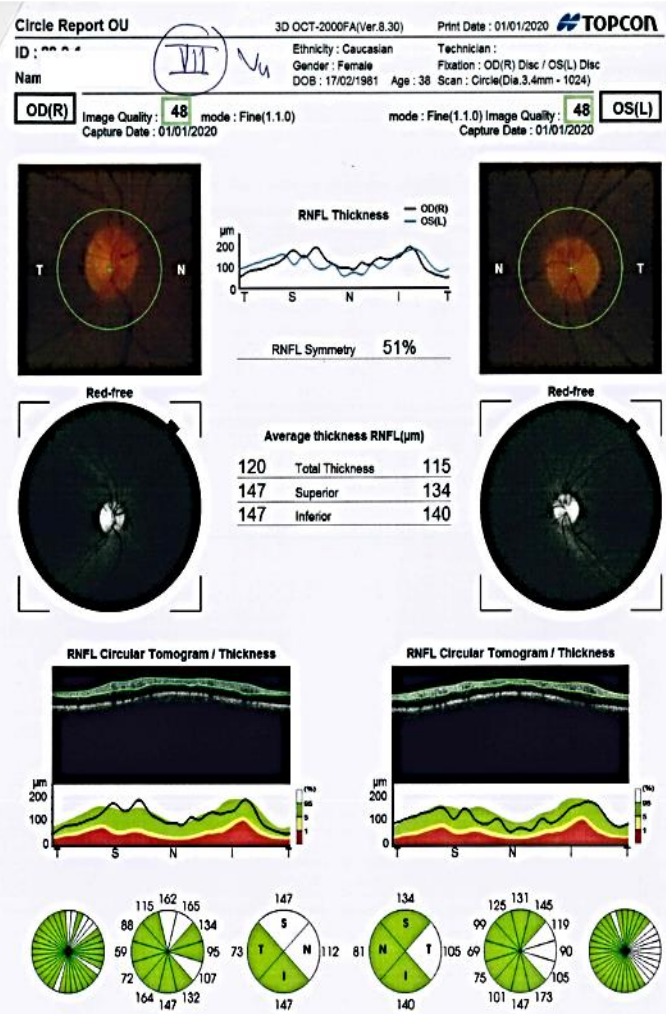

Cammens: Spposure Doso:
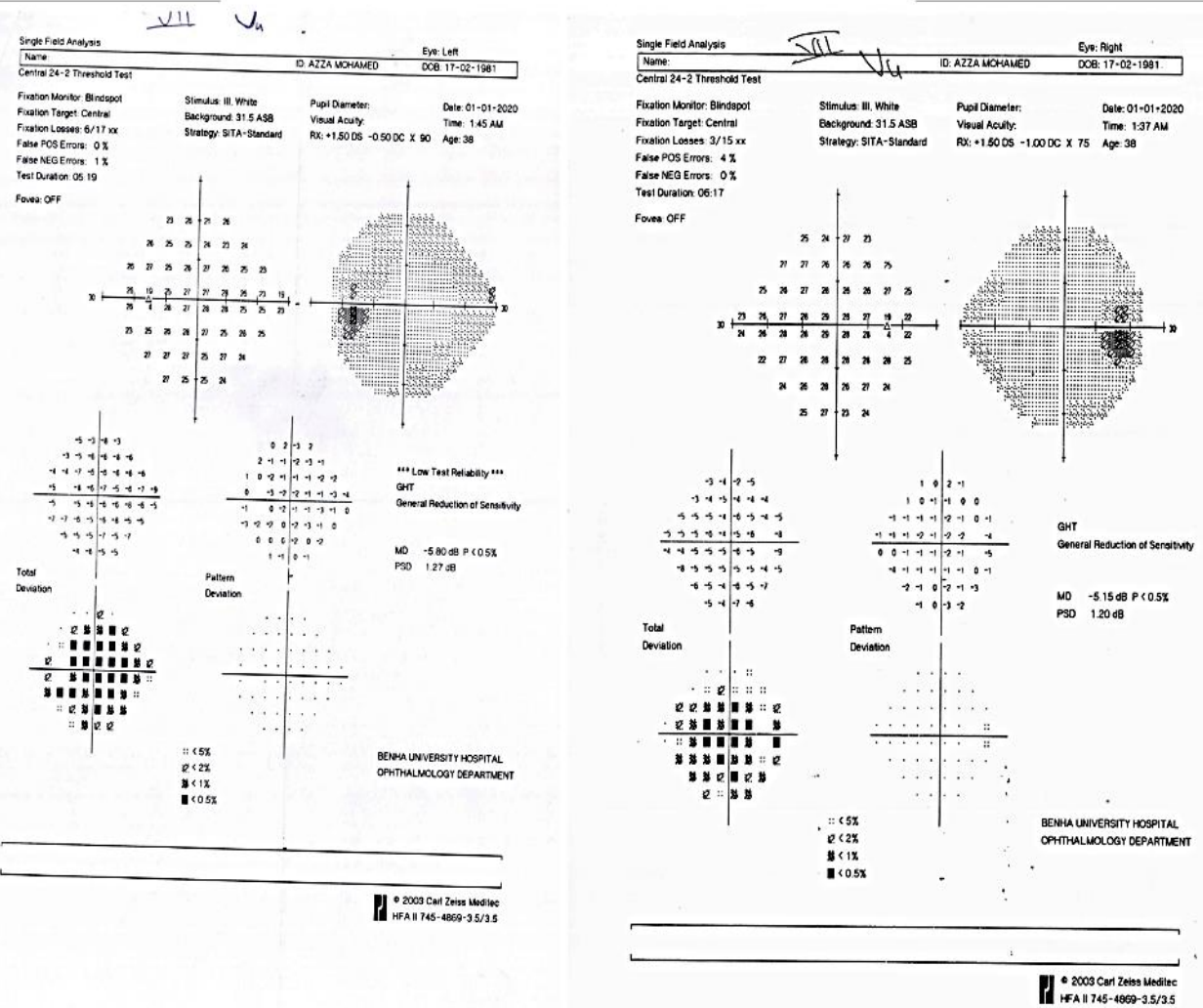

Fig. (8) OCT scan and VF test results of the fourth visit of patient number 7 in our study. Right eye: papilledema grade: 1 . RNFL thickness: $120 \mu$, Visual field finding MD $=-5.15 \mathrm{~dB}$. Left eye: papilledema grade 1 . RNFL thickness: $115 \mu$. Visual field finding $\mathrm{MD}=-5.80 \mathrm{~dB}$. 

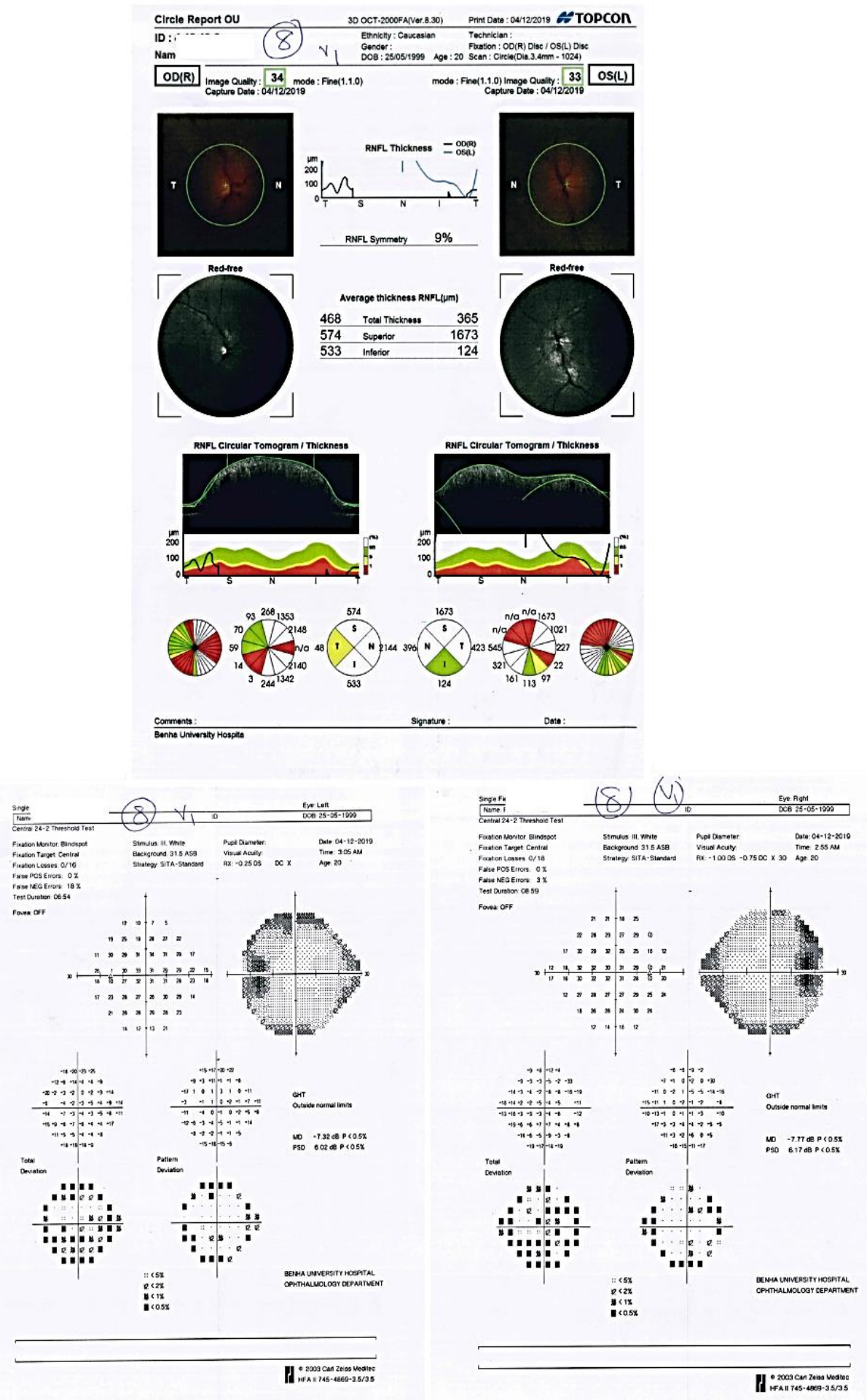

Fig. (9) OCT scan and VF test results of the first visit of patient number 8 in our study. Right eye: papilledema grade: 3. RNFL thickness: $259 \mu$, Visual field findings are EBS, nasal visual field defect, incomplete upper and complete lower arcuate scotomas, upper temporal field defect. $\mathrm{MD}=-4.74 \mathrm{~dB}$. Left eye: papilledema grade 3 .

RNFL thickness: $167 \mu$. Visual field findings are EBS and double arcuate scotomas. $\mathrm{MD}=-3.52 \mathrm{~dB}$. 


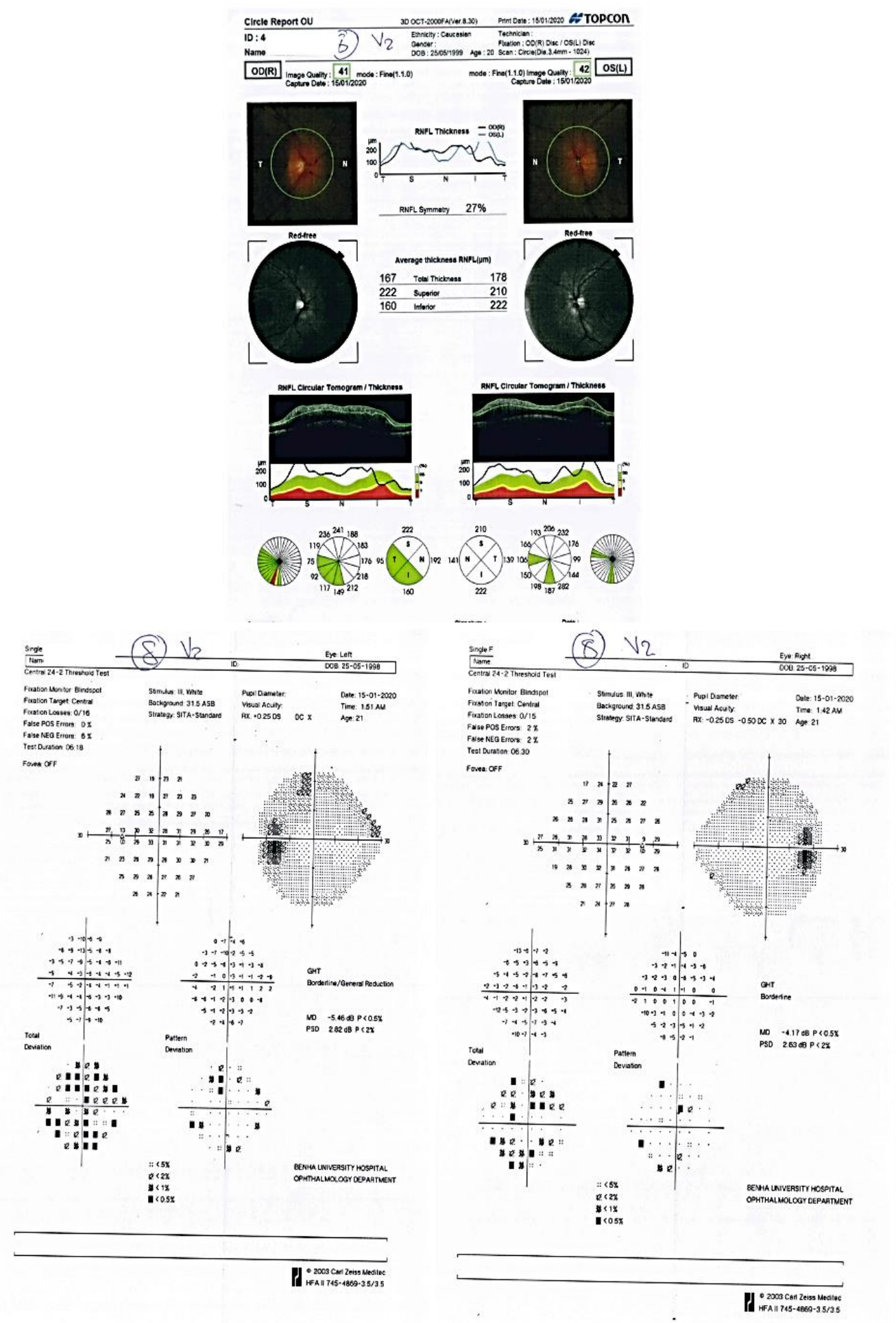

Fig. (10) OCT scan and VF test results of the second visit of patient number 8 in our study. Right eye: papilledema grade: 2 . RNFL thickness: $167 \mu$, Visual field findings are upper few scotomas and lower incomplete arcuate scotoma $\mathrm{MD}=-4.17 \mathrm{~dB}$. Left eye: papilledema grade 2 . RNFL thickness: $178 \mu$. Visual field findings are EBS and incomplete double arcuate scotomas. $\mathrm{MD}=-5.46 \mathrm{~dB}$. 

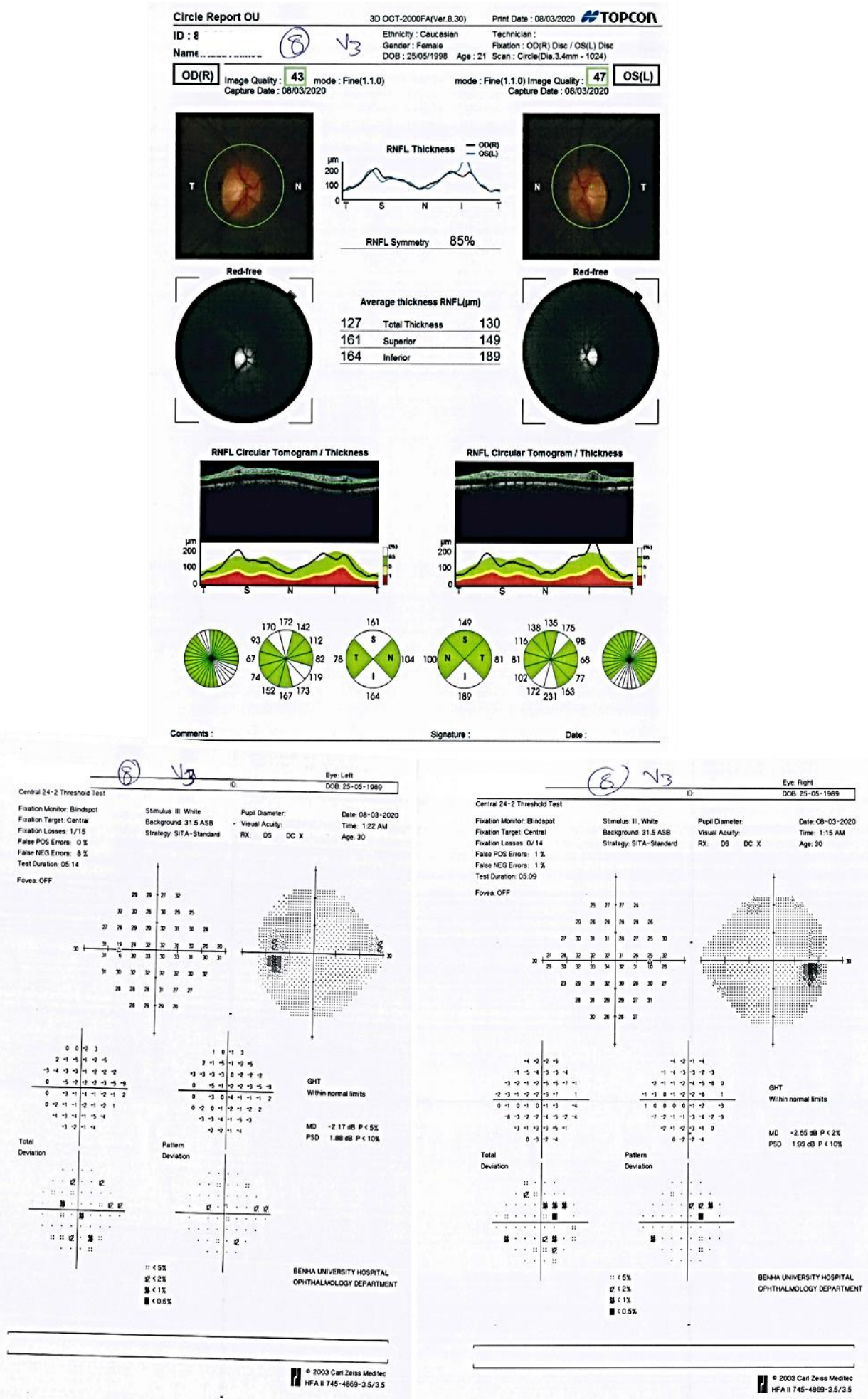

Fig. (11) OCT scan and VF test results of the third visit of patient number 8 in our study. Right eye: papilledema grade: 1 . RNFL thickness: $127 \mu$, Visual field finding is EBS. MD $=-2.65 \mathrm{~dB}$. Left eye: papilledema grade 1 . RNFL thickness: $130 \mu$. Visual field findings are few scattered scotomas. $\mathrm{MD}=-2.17 \mathrm{~dB}$. 


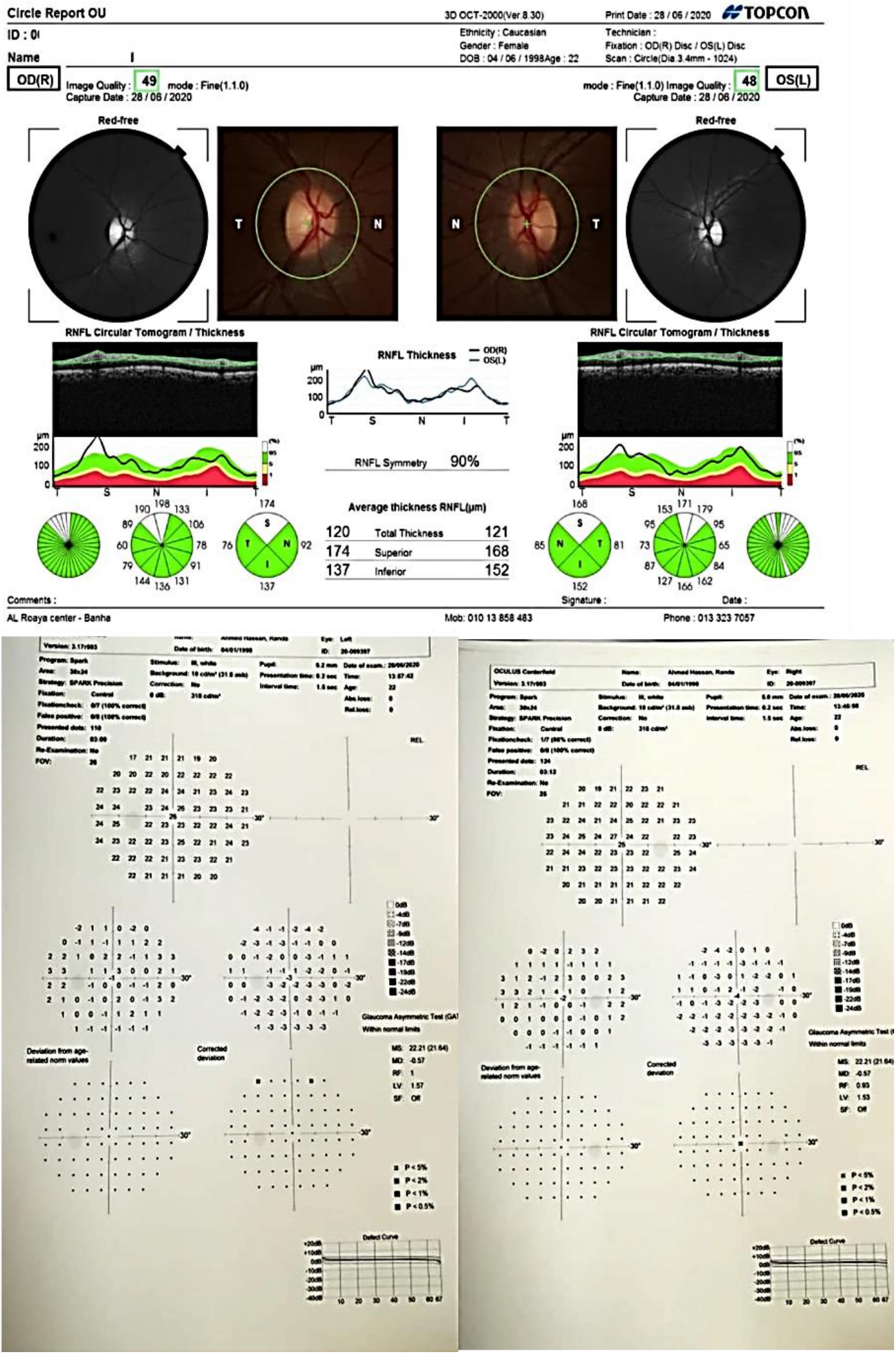

Fig. (12) OCT scan and VF test results of the fourth visit of patient number 8 in our study. Right eye: papilledema grade: 0 . RNFL thickness: $120 \mu$, Visual field finding is $\mathrm{MD}=-0.50 \mathrm{~dB}$. Left eye: papilledema grade 0 . RNFL thickness: $121 \mu$. Visual field finding is. $\mathrm{MD}=-0.57 \mathrm{~dB}$. 
Regarding medical treatment, it was started for all patients once diagnosed. Carbonic anhydrase inhibitor (CAI) was prescribed to all the 20 patients. CAI was given as a single medication in 9 patients (45\%). Both CAI and diuretics (Lasix) were prescribed for six patients $(30 \%)$ and five patients (25\%) were treated with CAI along with a short course of systemic steroids (Prednisolone tablets).

Potassium supplement tablets were given to ease the side effects of CAI.

Four patients reported being pregnant between the third and the fourth visit; hence CAI was stopped and only steroids were prescribed. No steroids were given to patients with high BMI. No patients had any other contraindications or known allergies to the prescribed medications. Analgesic tablets were prescribed only when necessary.

Lumbar puncture, along with medical treatment, was indicated in thirteen patients $(65 \%)$ out of the total 20 patients. Six patients were subjected to a single lumbar puncture procedure, five patients had two lumbar puncture procedures, one patient had three LP and one patient had five LP. A total of 24 lumbar puncture procedures were carried out throughout the study. Nine $(37.5 \%)$ of them were performed between the first and second follow up visits, twelve $(50 \%)$ were performed between the second and third visits and three procedures (12.5\%) were performed between the third and fourth (last) visit. Of note, a total of 21 LPs $(87.5 \%)$ were done before the third follow up visit as compared to three LPs done from $3^{\text {rd }}$ to $6^{\text {th }}$ month. Probably this can explain the significant correlation between the number of LPs and the PMD at the third visit.

Regarding the side effects to treatment, ten patients reported limb numbness; five of them also reported GIT upset. One patient reported excessive fatigue. All side effects to medications were well tolerated hence no medications were stopped due to their side effects. No patients showed any signs of drug related allergy. Six patients did not report any side effects to medications. All patients who underwent LP reported temporary back pain and local discomfort at the site of the lumbar puncture. Three patients reported post-LP headaches. No post-LP infection was reported.

\section{Discussion}

IIH is a relatively uncommon disorder with an average annual incidence of about one to two cases per 100,000 people in the general population. Obesity $(\mathrm{BMI} \geq 30)$ and female gender are the most frequently encountered risk factors of this disorder. Regarding the assessment of IIH, several studies have shown that quantitative perimetry and optic nerve examination are more sensitive than checking BCVA in the assessment of IIH. The presence of papilledema is the hallmark of suspected disease, and its severity determines the overall treatment strategy.
Papilledema can be monitored using both qualitative and quantitative methods. [1,2]

In our study, although we aimed to recruit patients regardless of their gender, three male patients were excluded from the very early visits of the study due to non-commitment to attend visits on the planned dates. We therefore included 40 eyes of 20 female patients. The mean age was $27.55 \pm 8.4$ years. All cases presented with newly diagnosed papilledema due to first reported episode of IIH. The diagnosis of IIH was made after all other causes of optic disc swelling were excluded and brain imaging was found free, accelerated hypertension was excluded by checking blood pressure for all patients.

Along with evaluation of the overall improvement of patients in our study, we investigated the correlation between papilledema grade, RNFL thickness measured by SD-OCT and the perimetric mean deviation (PMD) measured by automated perimetry. We also checked for any correlation between BCVA, BMI, age, modalities of treatment and any of the above mentioned investigations.

The first visit was the baseline visit; we started the treatment for all patients at the $1^{\text {st }}$ visit and followed them up at one month ( $2^{\text {nd }}$ visit), three months ( $3^{\text {rd }}$ visit) and six months (end-of-study visit). All patients underwent ophthalmic examination and investigations once diagnosed, including BCVA, color vision test, pupillary response, slit lamp examination, intraocular pressure check, ocular motility, confrontation test, and fundus examination. Ophthalmic investigations included measurement of peripapillary RNFL thickness by SD-OCT and visual field examination using automated perimetry. Treatment plan was determined, and treatment was given once diagnosis was made, supervised by both ophthalmologists and neurologists. Examination of the enrolled patients was re-done after one month, three months and six months.

We did not include patients with advanced disc swelling or chronic papilledema with postpapilledema optic atrophy, because irreversible axonal damage could hinder the correlation between disc morphology and perimetry.

In terms of clinical picture, at presentation, all patients experienced symptoms which were consistent with the diagnosis of increased intracranial tension (ICT). The main presenting symptom was headache in 14 patients (70\%), vomiting (15\%), tinnitus (30\%), vertigo (10\%) and visual symptoms in the form of blurriness with transient visual obscurations (5\%). No patients had marked visual loss or diplopia at presentation. In a study by Takkar et al., 2018 headache was reported by $85 \%$ of patients, Tinnitus in $52 \%$ of patients. Also, $22.5 \%$ of the patients in their study had diplopia at time of presentation and $25 \%$ had significant visual deficits at presentation. Our study did not find any significant visual deficits at presentation. That is most probably due to early reporting of symptoms, early diagnosis 
and management of IIH, as well as good patient compliance along the follow up period. Patients without headache in Takkar's study may have presented later after visual deficits had already taken place. [3]

Four patients $(20 \%)$ were already on OCPs at the start of the study. Three patients were known to have high $\mathrm{BMI} \geq 30$. No patients had any significant past medical, ocular or surgical history prior to the study. In our study, three patients (15\%) were found having a BMI $\geq 30$, we found no correlation between obesity and visual acuity outcome. Similarly, in the study by Takkar 2018, 10\% of patients were obese and BMI did not show any correlation with the final visual outcome. [3] No patients reported previous attacks of $\mathrm{IIH}$, no patients were suffering from any other systemic or ocular diseases. No patients were smokers or ex-smokers, and no one reported any history of known drug hypersensitivity reactions.

In a study done by Khin et al., they included patients with history of intake of OCPs and compared them with control group who didn't take OCPs. They concluded that OCP and other hormonal contraceptives were not significantly associated with a higher incidence of $\mathrm{IIH}$, arguing against the need for women with IIH to discontinue their use. [4]

Regarding visual acuity in our study, the median LogMAR BCVA was $0.00(0-0.2)$ at presentation, at the last follow up visit the median LogMAR BCVA remained the same $0.00(0-0.2)$. No patients experienced vision loss and no cases had optic atrophy along the study period. This can be due to early diagnosis and good patient compliance.

A study done by Gospe SM and colleagues, found that papilledema of modified Frisén grade $\geq 3$ on presentation was highly predictive of permanent vision loss $(\mathrm{P}<0.001)$, SD-OCT revealed optic atrophy in $13 \%$ and photoreceptor loss in $19 \%$ of eyes, with both findings highly associated with vision loss $(\mathrm{p}<0.0001)$.[5]

Several studies in literature found contradictory results about the correlations between grade of papilledema, BCVA and VF loss. Rush JA found no significant correlation between BCVA and field perimetry by Goldmann perimeter in IIH patients, while Orcut et al, found a qualitative correlation in atrophic or high-grade papilledema using the same device. $^{[6],[7]}$

In contrast, Göbel et al, did not find any quantitative correlation between disc swelling measured by HRT and automated visual field sensitivity.[8]

More recently, Mulholland et al, reported that, in individual patients with IIH, changes in confocal scanning laser ophthalmoscope (CSLO) disc volume were qualitatively correlated with corresponding perimetric sensitivity changes in the short term. However, no quantitative correlations were reported, either in cross-sectional or longitudinal measurements [9]. Moreover, Wall and White, while evaluating patients with IIH who had asymmetric papilledema, found a significant correlation between visual field sensitivity loss and papilledema grade, evaluated on optic disc photographs according to the Frisén scheme.[10]

Grading of papilledema in our study was carried out at each visit and recorded as each eye separately. In terms of laterality, we did not find any statistically significant relationship or difference between the findings between both eyes. Keltner and colleagues found that the left eye was the most damage eye (62\% of cases). They were not certain of the reason for this, but preferred sleeping position has been found to correlate with the presence of greater visual field loss (determined by a worse PMD) in glaucoma. It has been suggested that the left sided sleeping preference promotes gastric emptying and less gastric discomfort that might be important in obesity. ${ }^{[12]}$ Papilledema grade showed a significant correlation with both RNFL thickness $(r=0.590, \mathrm{p}<0.001)$ and PMD $(r=-0.205, \quad p=0.013)$ and insignificant correlation with LogMAR VA $(r=0.143, p=0.217)$

Regarding the overall improvement of the clinical picture and the investigations. At presentation, Frisén grade of papilledema ranged from grade 1 to grade 4 with median grade 3 . At the first month, it declined to grade 2 (range 1-3). Then declined further to grade 1 at the third-month visit (range 1-2) and at the last visit it declined to grade 0 (range 0-1). There was a significant decline of papilledema grade with treatment along the successive visits $(\mathrm{p}<0.001)$. No patients in the study showed worsening of papilledema grade through the follow up period. This could be due to close follow up and good patient compliance. Similarly, Frisén papilledema grading was also utilized in the IIHTT done by the Neuro-Ophthalmology Research Disease Investigator Consortium (NORDIC) which evaluated 6 months of acetazolamide and diet control for mild visual loss in IIH. Significant improvement in Frisén grade in both the study eye and the fellow eye were noted in fundus photographs. [11]

The most common visual field defects in our study were EBS with or without arcuate defects. Nasal field defect was the least defect seen. Similarly, in a study done by Keltner and colleagues, EBS with partial arcuate defect was the most common type found. There was a higher occurrence of visual field defects in the inferior hemifield and greater visual field loss in the left eye. Prior studies found that nerve fiber bundle-like defects, similar to glaucoma, are characteristic for optic nerve damage from increased intracranial pressure (ICP).[12] We found a significant correlation between Frisén grade of papilledema, and PMD ( $\mathrm{p}=0.013)$. Similarly, many studies evaluated patients with IIH and papilledema, reported a significant correlation between visual field sensitivity loss and papilledema grade after assessing optic disc photographs. [13, 14] 
Bidot and colleagues, studied the asymmetric papilledema that can arise in $\mathrm{IIH}$, they found that visual fields were worse on the side of the highestgrade papilledema $(\mathrm{p}=0.02)$. [15]

We found a significant correlation between papilledema grade and RNFL thickness along the study $(p<0.0001)$. Both values showed statistically significant overall improvement along the study. The mean total RNFL thickness was found to decrease along with the reduction of papilledema grade. The mean total RNFL thickness was $131.16 \pm 25.50 \mu$, $121.57 \pm 14.20 \mu, 120.3 \pm 14.7 \mu$ in the 1 st, 3rd and 6th months respectively $(\mathrm{p}<0.0001)$, while the median papilledema grade was 2 (1.5-2), 1 (1-2), 1 (0-1) at the same visits respectively. $(p=0.001)$. The same significant overall improvement applies to each eye separately.

Although Frisén grading of papilledema is subjective and dependent on experience, a study by Scott and colleagues reported a positive correlation between Frisén grading of optic nerve photographs and OCT analysis of optic nerves. In their study Frisén grading was found to be more reliable than OCT at higher grades of papilledema.[16]

It should be noted that the main problem using OCT as a single tool to follow up papilledema patients is that the reduction in RNFL thickness during treatment may result from two different mechanisms, the first is the actual resolution of edema. The second mechanism is the loss of nerve fibers. If nerve fibers are lost, a corresponding defect in field perimetry - as a functional test - will show permanent defects. That is why both OCT and field perimetry are mandatory to give simultaneous qualitative and quantitative assessment.[17] In our study, patients who showed a reduction of RNFL thickness did not show a corresponding visual field deterioration, which indicates a resolution of nerve edema rather than an atrophy of RNFL. Moreover, in some cases, when optic disc edema resolves, axonal damage has had already occurred but cannot yet be detected anatomically. At this time, a discrepancy between OCT and visual field testing can be helpful. In a study done by Rebolleda G. and colleagues., three eyes that developed irreversible visual field constriction, improved initially in OCT but not in visual field testing; indicating that at least in these three cases, RNFL attrition and swelling could occur at the same time. The tempo of visual loss may be rapid or slowly progressive, and so it is necessary to wait until the RNFL loss has reached a plateau to establish by OCT that irreversible damage has occurred.[18] However, in our study, no cases were reported having this problem. This may be due to the recent onset of IIH and because we evaluated patients with the first episode of $\mathrm{IIH}$, and no recurrences occurred during the study.

It is difficult to detect optic neuropathy in $\mathrm{IIH}$ because papilledema obscures the nerve making optic disc pallor difficult to be detected and prevents accurate assessment of axonal loss by OCT due to the significant swollen axons of the optic nerve.[16] However, in our study, VA showed a stable result throughout the clinical visits and visual field showed an improvement which denotes that no patients suffered optic atrophy.

To determine whether there was a loss of axons after papilledema subsides, Auinger and colleagues found that measurement of RGC layer thickness can be beneficial. They stated that in IIH, where continued papilledema can obscure OCT demonstration of RNFL thinning or atrophy, retinal ganglion cell (RGC) layer thickness measurement with 3-D segmentation, is a reliable structural biomarker of neuronal loss [19]

Savini G. and colleagues [20], in 2006 used OCT to detect and quantify RNFL thickness in patients with optic disc edema. They found diffuse thickening of the RNFL. Compared with eyes in a control group of 75 healthy subjects, eyes with optic disc edema showed a significant increase in the mean RNFL thickness in all quadrants (temporal, $\mathrm{p}=0.002$; superior, $\mathrm{p}<0.001$; nasal, $\mathrm{p}<0.001$; and inferior, $\mathrm{p}<0.001$ ). In patients who were followed up, progressive thinning was observed as the disease evolved toward either optic atrophy or clinical resolution. In our study, we observed a progressive reduction in RNFL thickness as the papilledema was subsiding, but no cases had optic atrophy.

We found an insignificant correlation in the study while comparing the total data of RNFL thickness and PMD ( $\mathrm{p}=0.627)$. However, the correlation between RNFL thickness and PMD was significant in the last visit of the study only $(p=0.04)$. We clinically noted an improvement of the grade of papilledema at the last visit, which tended to take a slower course of improvement when in contrary of fast changing PMD. That is probably due to the longer time the optic disc takes for the disc edema to resolve even after the resolution of clinical symptoms and PMD. This correlation at the last visit may demonstrate that the longer the patients are followed up, the more the correlation between PMD and RNFL thickness becomes more evident. Some studies that had a longer follow up periods than ours showed a positive correlation between PMD and RNFL thickness. This is one of the limitations of our study. Also, the frequent follow up of our patients when they were recently diagnosed (baseline, one month and 3 months visits) were less useful to demonstrate the correlation as compared to the visit with longer period (the last visit). Therefore, probably an overall longer duration of the study, with a wider interval of follow up would have been a better tool to detect the correlation.

The correlation between RNFL thickness and papilledema grade was found significant ( $\mathrm{p}<0.0001)$. Similarly, Salgarello T. and colleagues [21] studied eight patients with IIH and reported that disc volume changes correlated with corresponding PMD, and 
resolution of the papilledema coincided with improved visual field defects. Our findings in the present study strongly support these observations. Moreover, we found that the correlation between papilledema grade and RNFL thickness $(\mathrm{r}=0.590)$ was stronger than the correlation between papilledema grade and PMD ( $\mathrm{r}=-0.205)$ as shown by correlation coefficient (r).

A study done by Rebolleda and colleagues showed that peripapillary RNFL thickness abnormalities assessed by OCT in patients with mild papilledema was quantitatively correlated with visual field sensitivity losses. Their data support the possible use of OCT as a non-invasive quantitative method of monitoring the amount and evolution of papilledema. [18]

In the study of Rebolleda G. and colleagues [18], At the 1-year visit, 31.8\% of the eyes had an RNFL thickness that was higher in at least one quadrant compared with that of the normal database, indicating unresolved optic disc edema. Although they expected these percentages to change with longer follow-up, their data supports that over 12 months, the improvement in automated perimetry and the changes in OCT measurements correlated significantly. Our study followed up the patients for six months only, the values of RNFL thickness and PMD were heading towards normal at the end of study. The significant correlation between PMD and RNFL thickness was demonstrated at the end of our study. Probably a longer follow up period can show persistence of significant correlation. That is one of our study limitations.

We found no correlation between any of patient's age, laterality of the eye, oral contraceptive pills (OCP) intake or body mass index (BMI) and any of the study findings including best corrected visual acuity (BCVA), Frisén grade of papilledema, PMD or RNFL thickness. Keltner and colleagues found no differences in visual field results when analyzing by eye laterality or by patient's age. [12]

Concerning the treatment of IIH, all 20 patients received medical treatment at the first visit in the form of acetazolamide tablets along with oral potassium supplement (Slow-K). Frusemide diuretics (as Lasix tablets) were added when necessary. Some patients were started on short course of steroids to ease their symptoms. There were no contraindications to any medication and no drug allergy reported throughout the study. All patients showed marked improvement on medical treatment which is like IIHTT. [11]

Patients who complained from side effects as tingling and numbness reported that they were bearable and well tolerated. No medications were stopped because of side effects.

Lumbar puncture procedure was performed in 13 patients. Comparing the group of patients who had both LP and medical therapy (13 patients) to the other group that received medical therapy only (7 patients), we found no statistically significant difference in papilledema grade, PMD or RNFL thickness along the follow up period.

Clinically, it was noted that patients who received both medical treatment and lumbar puncture showed earlier resolution of clinical symptoms as headache, vomiting and tinnitus than patients who received medical therapy only. Similarly, in a study by Yiangou et al., (2018) [22] $71 \%$ of patients' symptoms improved at some point in the week postLP, with the greatest reduction in headache severity occurring at 1-hour post-LP. However, the comparison between both groups in terms of RNFL thickness and PMD improvement was statistically insignificant.

Regarding the correlation between LP times and LogMAR BCVA, papilledema grade, RNFL thickness and PMD, there was a significant correlation between the number of LPs and PMD of the third visit in both right $(p=0.039)$ and left eyes $(p=0.006)$. Probably that was due to the large number of LPs completed prior to the third visit. Twenty-one out of a total of 24 LPs have been already completed before the third visit. As a break down: 9 LPs were carried out between the 1st and 2nd visit and 12 LPs done between the 2 nd and $3 \mathrm{rd}$. After the patients had completed the third visit, there was a relatively longer period (of 3 months) till we reviewed the patients for a final end-of-study assessment (at 6th month). During the period between 3rd and 6th month, only 3 LPS were carried out. Overall, the higher frequency of LPs procedures was not significantly correlated to the improvement of any of the BCVA, RNFL thickness, or papilledema grade. Probably because of several reasons. Firstly, the VA remained stable throughout the study. Secondly, the optic disc swelling does not resolve immediately after the LPs, therefore it was difficult to link the papilledema grade of a certain visit to its corresponding LPs, also given that the recurrence of symptoms that necessitated more than one LP in between successive visits. Our patients did not necessarily receive equal number of LPs between each visit. Thirdly, because the neurologist's decision to go for some of the LPs in the study was based solely on the symptoms rather than on the ophthalmologist's feedback and grading of papilledema which, for example, was the case in the 3-month-period between the 3rd and the last visits when the patients were visiting the neurologist for a review but not regularly evaluated by an ophthalmologist.

Our study has few limitations; the first is the small number of patients enrolled in the study. The second is the gender being only females due to exclusion of three males for non-commitment to attend the planned follow up visits. That did not help us demonstrate the gender variation in response to medications, LP, morphological and functional changes optic discs. 
The third limitation is the relatively short follow up period as compared to similar studies. Longer follow up period might be required to demonstrate the correlation between RNFL thickness and PMD, to search for RNFL loss after resolution of papilledema. To appreciate the perimetric changes and to correlate with RNFL thickness. Also, to find a correlation after optic disc swelling begins to subside. Studies have demonstrated that in patients with resolved papilledema (and without relapse), there was a significant reduction in retinal thickness and RNFL at final follow-up than in healthy controls, possibly due to neuroaxonal damage and loss secondary to prolonged periods of raised ICP (Yri H. et al., 2012).[23] In our study, patients with chronic papilledema were excluded, no patients had recurrences, no patients had optic atrophy and all patients improved on treatment.

\section{References}

[1] DI.Friedman Idiopathic intracranial hypertension. Curr Pain Headache Rep.vol.11,pp.62-68,2007.

[2] JG.Heckmann , F.Faschingbauer, C.Lang Laser scanning tomography measurement of the extent of papilledema in the follow-up examination of patients with idiopathic intracranial

hypertension. Neurosurgery.vol.107,pp.543$547,2007$.

[3] A.Takkar, M.Goyal R.Bansal Clinical and Neuro-ophthalmologic Predictors of Visual Outcome in Idiopathic Intracranial Hypertension. NeuroOphthalmology.vol.42,pp.1-8,2018.

[4] P.Khin Kilgore, S.Michael Lee, A.Jacqueline Leavitt. A Population-Based, Case-Control Evaluation of the Association Between Hormonal Contraceptives and Idiopathic Intracranial Hypertension, American Journal of Ophthalmology.vol.197,pp.74-79,2019.

[5] SM III.Gospe, MT.Bhatti, MA.El-Dairi Anatomic and visual function outcomes in paediatric idiopathic intracranial hypertension. $\mathrm{Br}$ Ophthalmol.vol.100,pp.505-9,2016.

[6] JA.Rush Pseudotumor cerebri: clinical profile and visual outcome in 63 patients. Mayo Clin Proc.vol.55,pp.541$546,1980$.

[7] JC.Orcutt, NG.Page, MD.Sanders Factors affecting visual loss in benign intracranial hypertension. Ophthalmology.vol.91,pp.1303-12,1984.

[8] W.Göbel, WE.Lieb, H-J.Grein Quantitative and objective follow-up of papillary swelling with the Heidelberg Retina tomograph. Ophthalmologe.vol.94,pp.673$677,1997$.
[9] DA.Mulholland, JJ.Craig, SJA.Rankin Use of scanning laser ophthalmoscopy to monitor papilloedema in idiopathic intracranial hypertension. $\mathrm{Br} \quad \mathrm{J}$ Ophthalmol.vol.82,pp.1301-1305,1998.

[10]M.Wall, WNII.White Asymmetric papilledema in idiopathic intracranial hypertension: prospective interocular comparison of sensory visual function. Invest Ophthalmol Vis Sci.vol. 39,pp.134-142,1998.

[11] M.Wall, MJ.Kupersmith, KD.Kieburtz. The idiopathic intracranial hypertension treatment trial: clinical profile at baseline. JAMA Neurol.vol.71,pp.693701,2014.

[12] JL.Keltner, CA.Johnson, KE.Cello. for the NORDIC Idiopathic Intracranial Hypertension Study Group. Baseline visual field findings in the Idiopathic Intracranial Hypertension Treatment Trial (IIHTT). Invest Ophthalmol Vis Sci.vol. 29,pp.32007,2014.

[13] M.Wall, D.George Idiopathic intracranial hypertension. A prospective study of 50 patients. Brain.vol.114,pp.155-80,1991.

[14] TJ.Smith, RS.Baker Perimetric findings in pseudotumor cerebri using automated techniques. Ophthalmology.vol.93,pp.887$894,1986$.

[15] S.Bidot, BB.Bruce, AM.Saindane. Asymmetric papilledema in idiopathic intracranial hypertension. J Neuroophthalmol.vol.35,pp.31-6,2015.

[16]CJ.Scott, RH.Kardon, AG.Lee. Diagnosis and grading of papilledema in patients with raised intracranial pressure using optical coherence tomography vs clinical expert assessment using a clinical staging scale. Arch Ophthalmol.vol.128,pp.705711,2010 .

[17]ST.Bassi, KP.Mohana Optical coherence tomography in papilledema and pseudopapilledema with and without optic nerve head drusen. Indian J Ophthalmol.vol.62,pp.1146-51,2014.

[18] G.Rebolleda, J.Francisco Muñoz-Negrete. Follow-up of Mild Papilledema in Idiopathic Intracranial Hypertension with Optical Coherence Tomography. Invest. Ophthalmol. Vis. Sci.vol.50,pp.51975200,2009.

[19]P.Auinger, M.Durbin, S.Feldon. OCT SubStudy Committee for NORDIC Idiopathic Intracranial Hypertension Study Group, Baseline OCT measurements in the idiopathic intracranial hypertension treatment trial, part I: quality control, comparisons, and variability. Invest 
Ophthalmol Vis Sci.vol. 55,pp.8180$8188,2014$.

[20] G.Savini

C.Bellusci

M.Carbonelli

.. Detection

and

quantification of retinal nerve fiber layer thickness in optic disc edema using Stratus OCT. Arch Ophthalmol.vol.124,pp.1111111,2006.

[21]T.Salgarello, B.Falsini, S.Tedesco. Correlation of optic nerve head tomography with visual field sensitivity in papilledema. Invest Ophthalmol Vis Sci.vol.42,pp.1487-1494,2001.

[22] A.Yiangou, J.Mitchell, K.Markey.Therapeutic lumbar puncture for headache in idiopathic intracranial hypertension: Minimal gain, is it worth the pain? Cephalalgia.vol.12,pp122-133,2018.

[23] HM.Yri, M.Wegener, B.Sander. Idiopathic intracranial hypertension is not benign: a long-term outcome study. J Neurol.vol.259,pp.886-94,2012. 